\title{
Closing Persistent Gaps in Knowledge About Edge Ecology
}

\author{
Leslie Ries $^{1}$ (D) $\cdot$ Shannon M. Murphy ${ }^{2} \cdot$ Gina M. Wimp ${ }^{1} \cdot$ Robert J. Fletcher $\mathbf{J r}^{3}$
}

Published online: 22 February 2017

(C) Springer International Publishing AG 2017

\begin{abstract}
Edge effects are one of the most extensively studied ecological phenomena of the past 100 years. Despite the stillcommon perception that edge effects are overly complex and idiosyncratic, we do know a lot about the mechanisms that underlie them. A major review from 2004 described four fundamental mechanisms that cause most edge patterns. In general, altered resource distributions due to often-predictable gradients in the edge environment as well as access to multiple resources across edges accounts for the fundamental differences between habitat edges and interiors. However, altered species interactions near edges are another fundamental component of edge ecology and remain difficult to predict. Here, we follow up on that 2004 review and show that the same four mechanisms described there remain sufficient to explain most edge responses, but we highlight some new developments. Notably, we find that edge studies have widened to include many new habitat types, a more diverse taxa and broader regions of the world. Yet, even recent studies were often not
\end{abstract}

This article is part of the Topical Collection on Effects of Landscape Structure on Conservation of Species and Biodiversity

Electronic supplementary material The online version of this article (doi:10.1007/s40823-017-0022-4) contains supplementary material, which is available to authorized users.

Leslie Ries

Leslie.Ries@georgetown.edu

1 Department of Biology, Georgetown University, 37th and O Streets, N.W., Washington, DC 20057-1229, USA

2 Department of Biological Sciences, University of Denver, Denver, CO 80208, USA

3 Department of Wildlife Ecology \& Conservation, 110 Newins-Ziegler Hall, University of Florida/IFAS, Gainesville, FL 32611, USA designed to quantify critical metrics such as the depth or magnitude of edge influence. Further, sample sizes and study design continue to contribute to spurious variability and make comparing results difficult. These problems make extrapolation to larger scales problematic and a lack of effective tools for extrapolation complicate this goal even further. We offer suggestions on a set of best practices for designing edge studies and propose that future research should shift more towards extrapolation across landscapes.

Keywords Fragmentation $\cdot$ Ecotone $\cdot$ Boundary $\cdot$ Landscape

\section{Introduction}

Responses to habitat edges are one of the most well-studied ecological phenomena of the past century. From the first introduction of the concept in the early 1900s [1] to an explosion of research starting in the mid-1970s, edges continue to generate tremendous research activity (Fig. 1). Yet, edges are classically known for a complex, seemingly idiosyncratic jumble of responses (Ries et al. 2004). Furthermore, edge "rules of thumb" such as higher predation or higher diversity in edge habitats continue to persist in the field even though they are refuted by a number of reviews and meta-analyses [2-6]. However, edges are critical to our understanding of species distributions across landscapes, especially fragmented ones, so it is vital that we understand how they influence ecological processes.

In 2004, a major review of the edge literature was published by Ries et al. [7] (hereafter, the "2004 review") that described the dominant mechanisms underlying edge effects and reviewed the consistency of patterns reported in the literature. The 2004 review demonstrated that it is indeed possible to make sense of variable edge responses by individual species 
a overview of the edge literature $2005-2015^{*}$

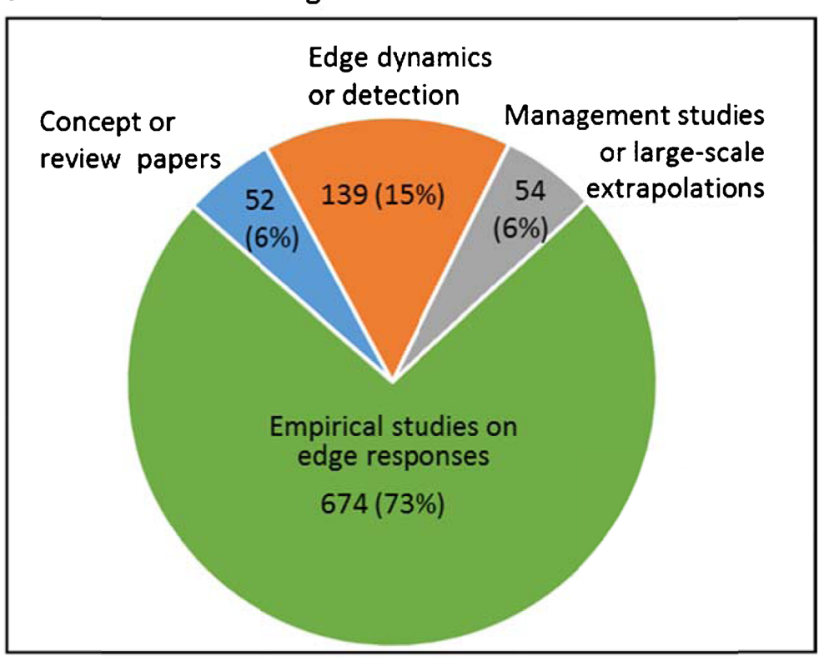

b yearly breakdown for empirical papers by topic

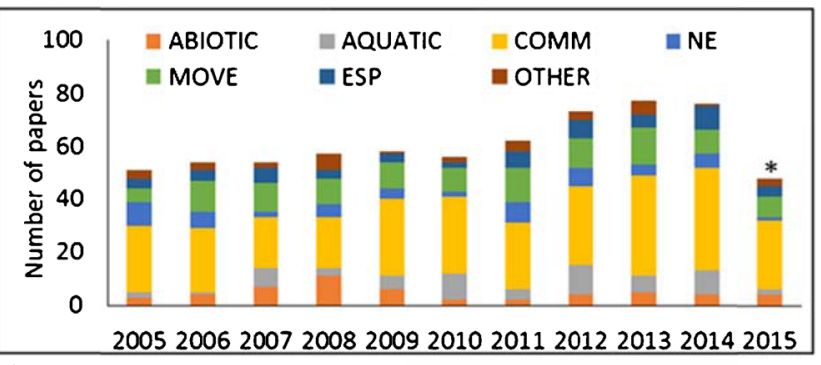

* Review through September 2015

Fig. 1 An overview of the amount and types of studies focused on habitat edges at the patch scale (not broad ecotones) between 2005 and 2015 (see Appendix 1 for search methods). We placed each of the 919 papers found into broad categories (a) and also show yearly publication rates by topic for empirical edge response studies (b). Topics include studies on: abiotic responses to edge (ABIOTIC), aquatic systems (AQUATIC), communities of terrestrial organisms (COMM), natural enemies (NE), movement (MOVE), ecosystem services and other ecological processes (ESP) and OTHER (responses of single species, multi-trophic interactions and evolution)

if their relationships with resources associated with both adjacent habitats were accounted for along with confounding factors such as edge orientation (e.g., north- vs. south-facing). However, the 2004 review also found that little progress had been made in quantifying how local edge gradients could be scaled up to understand larger patterns of distribution.

Here, we do a follow-up to the 2004 review by surveying the literature from the past 11 years. Our primary goal was to determine if the broad set of mechanisms presented in the 2004 review remained sufficient to understand recently reported responses and also to determine the extent to which criticisms from the 2004 review about study design and lack of scaling up had been addressed. To do this, we carried out a broad review of topics over the last 11 years and also a detailed review of the recent (2013-2015) empirical literature. We found a large number and many different types of studies published on edges over the past decade and placed them into broad categories by research focus (Fig. 1). To contend with the sheer volume of edge studies that are published yearly, our strategy for this broad overview was to focus on papers that present new conceptual approaches or those that performed in-depth reviews of select topics in the literature. Our goal was not to perform a meta-analysis of results since that has been done for several individual topics and instead we perform a review of those reviews. Detailed methods and references are provided in Appendix 1. The 2004 review made a strong claim that we can understand and account for much of the variability of edge responses, yet challenged the research community to do a better job of designing edge studies so that the results were more relevant to large-scale analyses. Our goals for this review were to follow up on those claims and see how far the research community had advanced those goals. We did that by answering the following questions:

1. How well do we understand the mechanisms underlying edge responses?

2. How well can we understand variability in edge responses?

3. How successful have we been at extrapolating edge responses to larger scales?

4. How should edge studies be designed to better elucidate mechanisms or be scalable to larger landscapes?

\section{How Well do we Understand Mechanisms Underlying Edge Responses?}

One of the main conclusions of the 2004 review is that we do have a strong understanding of the fundamental factors that alter ecology at habitat edges. The 2004 review summarized four fundamental mechanisms underlying edge responses, each of which had long been described in the edge literature. Two of these mechanisms are unique to edges: 1) ecological flows of materials, energy and organisms from one adjacent habitat into another and 2) access to complementary resources divided between adjacent habitats. The second two mechanisms are fundamental ecological processes (not special to edges) that nevertheless amplify ecological distinctness between edge and interior zones: 3 ) resource mapping of one species onto a gradient caused by another species responding to either of the first two edge mechanisms and 4) novel species interactions due to an altered community of organisms at the edge.

Despite a great deal of research over the past 11 years (Fig. 1), little has emerged from the empirical or conceptual literature to suggest that the above four mechanisms do not capture the vast majority of edge responses. This is true even as the scope of studies has expanded. For instance, almost $10 \%$ of studies were aquatic in nature during the last 11 years (Fig 1b), but rare before that. Nevertheless, the basic 
conceptual frameworks that were developed based on terrestrial systems seemed to transfer well [8]. Further, the geographic locations, focal taxa, and focal habitats continue to expand (Fig. 2a, b and 2c).

One mechanism that was not included in the 2004 review was edge geometry, specifically that just being near the physical boundary could impact how individuals are distributed near edges $[9,10]$. This issue is analogous to the middomain effect in biogeography, but operating within patches. For example, the territorial boundaries for a species may limit the number of individuals that could be packed near the edge relative to the interior, lowering the density even if there is no difference in habitat quality. At the time of the 2004 review, it was deemed not common or well-established enough to be considered as a general driver (even though it had been treated in a few studies, e.g., [9-11]), but three recent studies suggest that decision should be revisited. Prevedello et al. [12] developed a model to show that geometric edge effects should be common. Two additional modeling exercises developed null models of edge effects, essentially assuming no biological basis for avoiding or preferring edges, but still found emergent edge effects simply because the geometry of an edge can allow immigrants from all sides rather than just a portion of the surrounding landscape $[13,14]$. We suggest future researchers consider testing for this geometric constraint (or consider using this factor in null models) to see how useful these approaches are and whether we expect species with certain traits (e.g., territoriality) to exhibit geometric effects more frequently.

One factor that has been cause for confusion in edge studies is the cascading impact of direct and indirect responses [15]. Ultimately, most edge effects that are described are indirect inasmuch as species are usually responding to altered distributions of their resources, often driven by abiotic changes. This has led some researchers to try to isolate responses caused solely by the presence of edges from patterns that can be linked to measurable gradients with respect to edge distance (e.g., light levels, floristic changes, etc.). However, studies that attempt to determine if observed edge gradients are "really" edge effects, or are instead caused by some other underlying environmental gradient can be missing the point [15]. Even if some local environmental variable can be found that is the direct cause of an observed edge response (e.g.
Fig. 2 Description of study systems for empirical studies of edge responses from 2013-2015 (movement and aquatic studies were not included). For each study, we recorded study location (a), focal taxa (b), the types of edges studied and whether the studies took place on only one side of an edge or both sides (c), for one-sided edge studies (in forest only), what type of habitat was adjacent to the focal patch (d), and what response type was measured at the community, species, and process levels (e)

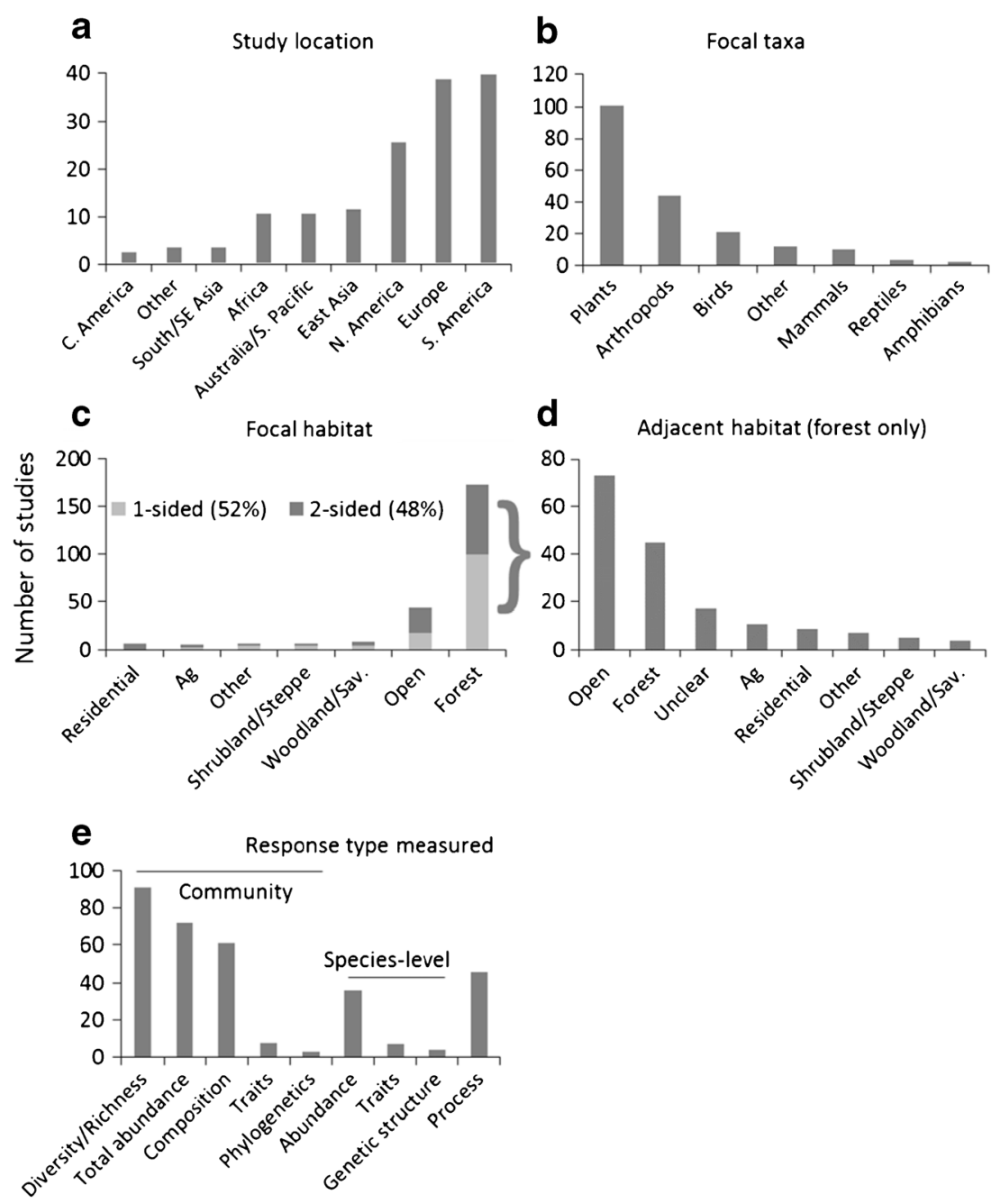


[16]), if that gradient itself is caused by edges, then the response is, ultimately, an edge response [15]. This is important because if there is an attempt to separate edge responses from edges, it becomes much more difficult to predict where those environmental gradients are likely to occur in space. Instead, if the connection is retained, then the presence of edges can serve as a powerful proxy for landscape-scale dynamics when it is known that an edge is linked with a typical environmental gradient and potentially a suite of associated responses.

Finally, it is important to note the advancement in the recent literature in terms of the broadening of topics studied (Figs. 1 and 2). In addition to studies of classic edge abundance patterns and critical factors like predation rates, we found studies increasingly focused on nutrient cycling, species interactions, and behavior (Fig. 1). While traditionally edge studies did not examine interactions for more than two trophic levels, at least two studies from our recent review studied multiple trophic levels, three levels in one case [17] and four in another [18]. Several studies also investigated the phylogenetic structure of the community (Fig. 2e) or small-scale genetic structuring that could be tied to edges (e.g., [19]). These studies are likely to deepen not only our understanding of edge response patterns but of macro-ecological or ecosystem-level dynamics.

\section{How Well can we Understand Variability in Edge Responses?}

One of the fundamental critiques put forward by the 2004 review was that the variable responses frequently reported in the edge literature could not be resolved without a conceptual framework to make sense of them. The 2004 review suggested that the best way to correct this is developing specific, directional predictions for expected responses (as opposed to just a classic alternative of "difference" to a null hypothesis) based on an a priori framework. The value in making directional predictions is that researchers are compelled to propose a specific mechanism and are better able to make sense of observed variability rather than trying to develop post-hoc explanations. Although patterns can easily be swamped by local effects when sample sizes are small $[20,21]$, understanding variable responses and their alignment with current edge theory also lays the groundwork for updating and improving the models when new generalizations can be made. Ultimately, one can make the claim that if you cannot predict something, you do not really understand it. Further, since we generally are interested in edge responses in terms of the role they play in fragmentation and landscape-scale dynamics, it is important to know how consistent responses are across time and space.

There are currently three conceptual frameworks (Fig. 3) of which we are aware that generate specific predictions about edge responses. These models allow researchers to make a priori predictions, either about the character of the responses
(Fig. 3a and b) or the species likely to be most impacted by edges (Fig. 3c). Despite the benefits of making a priori predictions, they were still used in only about $33 \%$ of the papers we reviewed; although this is still an improvement since the 2004 review when synthetic, predictive models had just been published. Most of these predictions are based on some knowledge of resource distribution, but some still use edge "rules of thumb", so we address the predictive power of each approach separately below. In all cases, "correct" means the predicted response was observed and "incorrect" means it was not. Note that all predictions are categorical and none are quantitative, so classification of correct and incorrect is straightforward.

\section{Predictions Based on Resources}

The only current conceptual framework for species-specific edge responses (i.e., predictions based on the biology of a specific organism and not traits) has been the resource-based model proposed by Ries and Sisk [25], hereafter called the edge resource model (ERM, Fig. 3a). This model was based on the first three mechanisms described above (ecological flows, access, resource mapping) and used those basic concepts to develop qualitative predictions of species abundance as a function of distance from edge. Briefly, species that are adjacent to lower-quality habitat with no complementary resources (resources not already found in their focal habitat) are predicted to decline near edges, although individuals may "spill over" into adjacent habitat. This is called a "transitional" edge response. In the case where habitat quality is equal in adjacent habitats, no edge response ("neutral response") is expected. Alternatively, species that are in adjacent habitats that each contained critical resources, but that were divided between adjacent habitats (complementary) are predicted to have their highest densities at the edge, so a positive edge response is expected on both sides of the edge.

The 2004 review used this as a framework to make qualitative predictions for observations reported in the literature and showed that the model did fairly well at predicting responses with some caveats (see below). Since the 2004 review, several papers have used the ERM as an organizing conceptual framework, but primarily to explain the patterns they observed post hoc, not to make a priori predictions. Further, predictions were often made not for individual species, but for communities that either shared some trait or were presumed to have similar resource requirements. For the one study that did make a priori predictions [18], the ERM successfully predicted the response of herbivores, mesopredators, and top predators to the habitat edge. However, the in-depth nature of the study suggested one of the assumptions of the underlying mechanisms may not be the actual driver. Specifically, although specialist herbivores avoided the edge, no difference in food quantity or quality could be found there, 
Fig. 3 Conceptual edge response models. The most commonly used model is the edge response model (a). This model predicts positive, neutral or negative edge responses based on relative habitat quality and whether resources are divided or not between habitat patches (reprinted by permission from [22]). An expansion of this model predicts response shape based on the strength of quality differences between habitat (b). Adapted with permission from [23]. Ewers and Didham [24] suggested how traits might be associated with different aspects of fragmentation including edges (c). Reprinted with permission by [24] a

Edge Response Model (ERM)

b Villasenor model extension

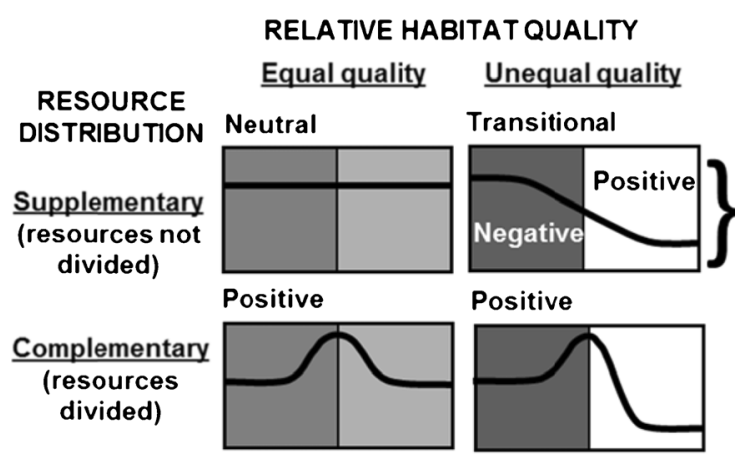

C

\section{Ewers and Didham trait-based model}

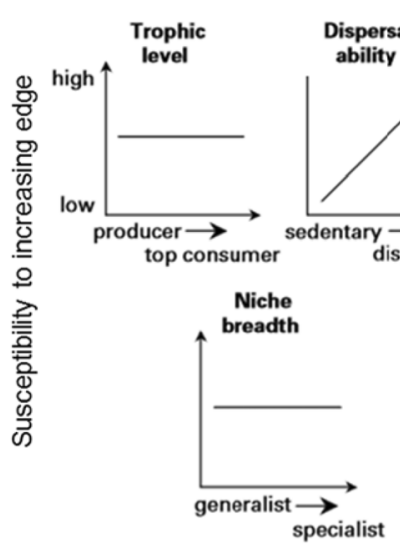

adjacent habitat neutral

Moderated spillover

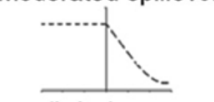

non-limited access or

high boundary permeability

Extended spillover

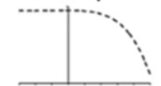

high emigration from higher quality or preferred habitat

adjacent habitat negative

Transitional

(moderated spillover)

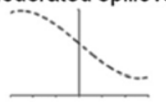

Negative

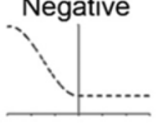

Rarity

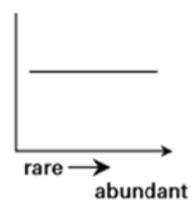

Extended negative

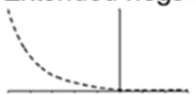

high sensitivity to habitat change (e.g. core-area species) and the authors suggested the herbivores may instead be avoiding predators that congregate at the edge [18].

For other studies that used the ERM to understand species responses post-hoc, the ERM largely explained species distribution patterns where the natural history of the organisms was well-understood (and this was also true in aquatic communities [8]). However, in systems where natural history and ecological information for the species of interest was scarce, the utility of the ERM in understanding species responses to the habitat edge was mixed [26]. Additionally, because the ERM is by definition a bottom-up resource-driven model, this model often failed to explain species responses to the habitat edge that were driven by predation [8].

While most studies that made a priori predictions did not specifically use the ERM, roughly half of these (23 out of 49 studies) used information on the distribution of critical resources in order to predict species, trait group or community responses to the habitat edge. From these 23 studies, the three possible edge response outcomes (negative, neutral, positive) were correctly predicted about $63 \%$ of the time ( 32 out of 51 results reported; note that a single study often reported more than one result). Many studies did not use a specific model or information about resources to make their predictions. For these 26 studies, the information on which these predictions were based came from another study system or the basis for prediction was unclear. Surprisingly, these studies did only slightly worse at predicting species' responses to the habitat edge; in these cases, observations matched predictions only about $53 \%$ of the time ( 25 out of 44 responses reported). For these, community-level predictions were better supported by data than single-species predictions.

Despite the overall low rates of correct predictions, when predictions were incorrect, a neutral response was usually observed where a significant edge response (either positive or negative) was predicted ( 15 out of 19 cases). This was similar to what was found in the 2004 review where unpredicted neutral responses were often observed, but almost never a response in the opposite direction of what was predicted. One possible explanation is that many edge response studies have extremely low replication and transects often do not extend very far into habitat interiors. This results in high levels of "neutral variability" (a mix of uni-directional responses with neutral responses); unfortunately, we have seen little improvement in study design to improve the power to detect edge responses (see the section on study design, below).

A biological reason why we may see neutral responses rather than predicted directional responses is that species may be differentially sensitive to edges [27]. This is only a 
potential explanation when a neutral response is observed when a directional one was predicted, but that accounts for the vast majority of prediction failures. The idea is that, for some species, even in conditions that suggest they should avoid or be attracted to edges based on a framework like the ERM, they never or rarely show edge responses, even where expected. For instance, even after controlling for distribution of resources that would predict edge responses, darker colored butterflies seemed to be less likely to show predicted responses compared to lighter ones [27], which suggests that darker butterflies may be less sensitive to edges. Ewers and Didham [24] presented a framework for understanding how species with different traits should respond to all types of fragmentation effects, although their conceptual framework (Fig. 3c) suggested that only dispersal ability should be related to edge sensitivity [28]. We suspect a much wider range of traits will be found to be important, but this area of research is still in its infancy. Ultimately, if certain species can be shown to consistently ignore edges regardless of the distribution of resources with respect to the edge, it may make sense to label those species "edge-neutral" and, further, to look for traits that may unite those species (and thus increase general predictability of edge responses).

We found only rare instances where observed responses were in the opposite direction of predicted responses or occurred when neutral responses were predicted ( 2 and 2 out of 19 cases, respectively). We believe that the continued rarity of these outcomes (they occurred in only $14 \%$ of cases in the 2004 review) shows that when edge responses are observed, they are often predictable based on resource distributions. Although top-down forces may also drive some patterns [18], when the presence of predators or parasitoids was used to predict responses to the habitat edge, discrepancies between predictions and observations were quite common [29, 30]. Incorporating top-down impacts into the ERM would be an improvement on the current model and should be a priority as a future research goal, but may prove challenging in execution given how poorly we still understand when predation is likely to be higher at edges $[2,4-6]$ and even whether heightened predation near edges can explain variation in species abundances given dynamics like ecological traps [31].

One drawback of the ERM is that it only makes qualitative predictions (positive, negative, or neutral), but a recent adaptation [23] of the ERM develops specific predictions about response shape based on how strong the quality difference is between adjoining habitats (Fig. 3b). As a relatively new mod$\mathrm{el}$, this has not yet been empirically tested and even in the primary paper where it was constructed it was developed post-hoc based on the patterns observed in their study system (mammals at urban forest edges). However, the goal of making edge response predictions more quantitative is an important one and we look forward to future tests of these predictions.

\section{Predictions Based on Edge "Rules of Thumb"}

The two most common edge "rules of thumb" are that 1) predation and 2) diversity are generally higher near edges. The idea that predation rates are higher near edges has been consistently debunked (see [2, 5, 6, 32] among many others). We do not address this in depth here except to say that only 3 of the 7 studies in our review of the recent literature that predicted higher predation rates at the edge actually showed higher predation rates. We examine the pattern of community richness more closely because that has not yet been thoroughly reviewed. The idea that edges are zones of higher diversity goes back to Leopold [33] and has two fundamental causes: 1) spillover of individuals from each of the abutting habitats into the adjacent habitat and 2) unique species associated with the edge. Although studies frequently assume higher diversity at edges based solely on this rule of thumb, in reality, diversity metrics tend to be variable. In our review, when authors made this prediction, diversity was higher at edges only $50 \%$ of the time ( 5 out of 10 cases). One possible cause is that edges might be assumed to be between two diverse habitats (e.g., forest and grassland), whereas many habitats now abut highly disturbed and degraded habitats; in these cases, a loss of diversity might be a more likely outcome [34]. Distinguishing between these situations in the future may lead to more accurate predictions of community richness near edges.

An alternate approach to making predictions at the community scale is to determine if there are shared resources used by the community, and, if so, then apply the principles of the ERM. While the ERM was specifically developed to describe single species responses to the habitat edge, studies that used the distribution of resources to predict species richness or diversity responses to the habitat edge were generally more successful in making correct predictions than those relying on rules of thumb. For instance, fungi generally require cool, moist habitats and, as expected, they tend to avoid forest edges as a group [35]. As noted previously, when studies incorporated knowledge of critical resources, they correctly predicted species richness and diversity responses around $64 \%$ of the time ( 9 out of 14 cases). This suggests that when there are common resources for a group, resource distribution may be a better predictor of community richness than relying on rules of thumb. However, this will only work when species within a clade share fundamental resources that tend to be associated with particular habitat types.

Finally, richness and diversity patterns often miss more subtle patterns that can be elucidated if actual community composition is considered. However, community structure is rarely quantified and, to date, no one has offered a conceptual framework for how community structure should change near edges. Although challenging, the goal of developing a true community-based predictive model is one that should be tackled. This can either be done through the development of novel 
theory relative to edges or by incorporating edge dynamics into current theory of community assembly (e.g., environmental filtering, assembly rules based on functional groups, neutral models, etc.).

\section{Interactions and Confounding Factors: Grappling With Variability in Edge Responses}

One of the main results of the 2004 review was to show that variability in edge responses was manageable and even predictable. The first step was to control for edge type and context, but even after that major source of variability was accounted for, much variability remained, but was of the "neutral" variety described above. Sometimes, responses were of different magnitudes (yet in the same response direction) or in some cases, a unidirectional response was mixed with some neutral responses, but a mix of positive and negative responses was rare ( $<2 \%$ of reported responses). In reality, this mix of variable-strength unidirectional and neutral responses may be partially due to differences in study design rather than differences in the actual ecology. However, there are many biological reasons why the strength of edge effects may differ between studies. For example, the 2004 review found that edge orientation consistently resulted in this "typical" range of responses (variable strength or neutral mixed with unidirectional responses, but rarely a direction switch). This makes sense since edge orientation tends to weaken or strengthen the abiotic gradient associated with edges dependent on sun position. Fragmentation had similar effects, with interactions with patch areas likely the main cause of underlying interactions - although few studies do a good job of disentangling these factors ([28]). Temporal differences were also found, although without determining underlying causes; so, the actual mechanism of that variability is undetermined but still "typical" in terms of how it manifests (weakening or loss of edge responses, not direction switches). Although in principle those temporal shifts may be due to shifting resources (or seasonal variation in resource needs), there has been little progress made showing that temporal variability is predictable (via, for example, putting the ERM into a temporal context). That type of demonstration would be helpful for increasing the predictability of edge responses across time and space.

Another potential cause of edge response variability is edge contrast; it is often assumed that responses should be stronger at "hard" rather than "soft" edges. The 2004 review found it difficult to test that prediction systematically because, in general, studies did not account for habitat quality, which was likely to be confounded with adjacent structural contrast. Of the few studies that did account for habitat quality differences, the results were mixed. Since that review, additional research has continued to show that hard edges tend to elicit stronger responses than soft edges $[23,36]$. However, without a priori controlling for habitat quality in adjacent habitat, it is still difficult to tease out the importance of physical edge contrast with the fact that similarly structured habitats often have more similar resource availability compared to starkly different habitat structures. Since most studies still fail to do this (but see [37]), we suggest that the role of edge contrast per se is still not wholly worked out but that may prove to be a useful proxy for underlying resource-driven dynamics.

Finally, we highlight what is likely one of the primary causes of variability in edge responses that was only alluded to in the 2004 review: variability in study design. We have already highlighted several times where variability of unpredicted neutral responses (or variable responses that are basically a weakening or loss of a unidirectional response) could be due to lack of statistical power. At the time of the 2004 review, studies were plagued with low sample size, variable transect length, and variable number of edge distances sampled. Our recent review shows that many studies continue to have low sample size and set a priori constraints on findings by having few sampling distances and highly variable "maximum" distances (Fig. 4), all of which are likely to lead to variable results. We suggest some basic guidelines in the final section of this paper.

\section{How Successful Have we Been at Extrapolating Edge Responses to Larger Scales?}

Edge effects have become such an important topic of research over the past century because they are a key to understanding distributions across landscapes, especially fragmented ones. Yet, the 2004 review pointed out that results are rarely used to extrapolate over larger scales, and this continues to be true with only $5 \%$ of studies since 2005 applying what we know about edges either to management or larger-scale extrapolations (Fig. 1). Further, the information necessary to effectively extrapolate edge responses is rarely reported in most studies, including understanding the depth and magnitude of edge influence (Fig. 4c) and accounting for multiple edge effects and complex geometry. Further, we still lack the tools to make it tractable to carry out these studies. Effectively, this omission means that, despite decades of research on edges, we rarely leverage edge data to better understand patterns across landscapes [38]. In this section, we review the progress in this area.

\section{Depth and Magnitude of Edge Influence}

Over the past decade, much progress has been made in the development of formal methods to estimate edge effects. Edge effects can be reduced into two components: the depth of edge influence (DEI) and the magnitude of edge influence (MEI). This distance is frequently used to distinguish 'edge' from 'interior' portions of patches and is integral to the use of core area models (see below). The magnitude of edge influence 
Fig. 4 Study design used for empirical studies of edge responses from 2013-2015 (movement and aquatic studies were not included). The maximum ( $\max$ ) distance study plots were placed from the edge varied not only in their distances but how uniform the max distance was and how precisely it was measured (a). For instance, sometimes only a minimum max distance was reported (e.g., somewhere beyond $100 \mathrm{~m}$ away from the edge, but not less). In other cases, a zone of distances was given (e.g., somewhere between 100 $200 \mathrm{~m}$ from the edge). Finally, sometimes a set max distance was used (e.g., $100 \mathrm{~m}$ from the edge). Number of distance classes also varied (b). Whether studies reported distance of edge influence (DEI), magnitude of edge influence (MEI), both or neither was also scored (c). Study design included replication in terms of number of "independent" sampling blocks (d) and the number of replicates per block (e). See Appendix 1 for details
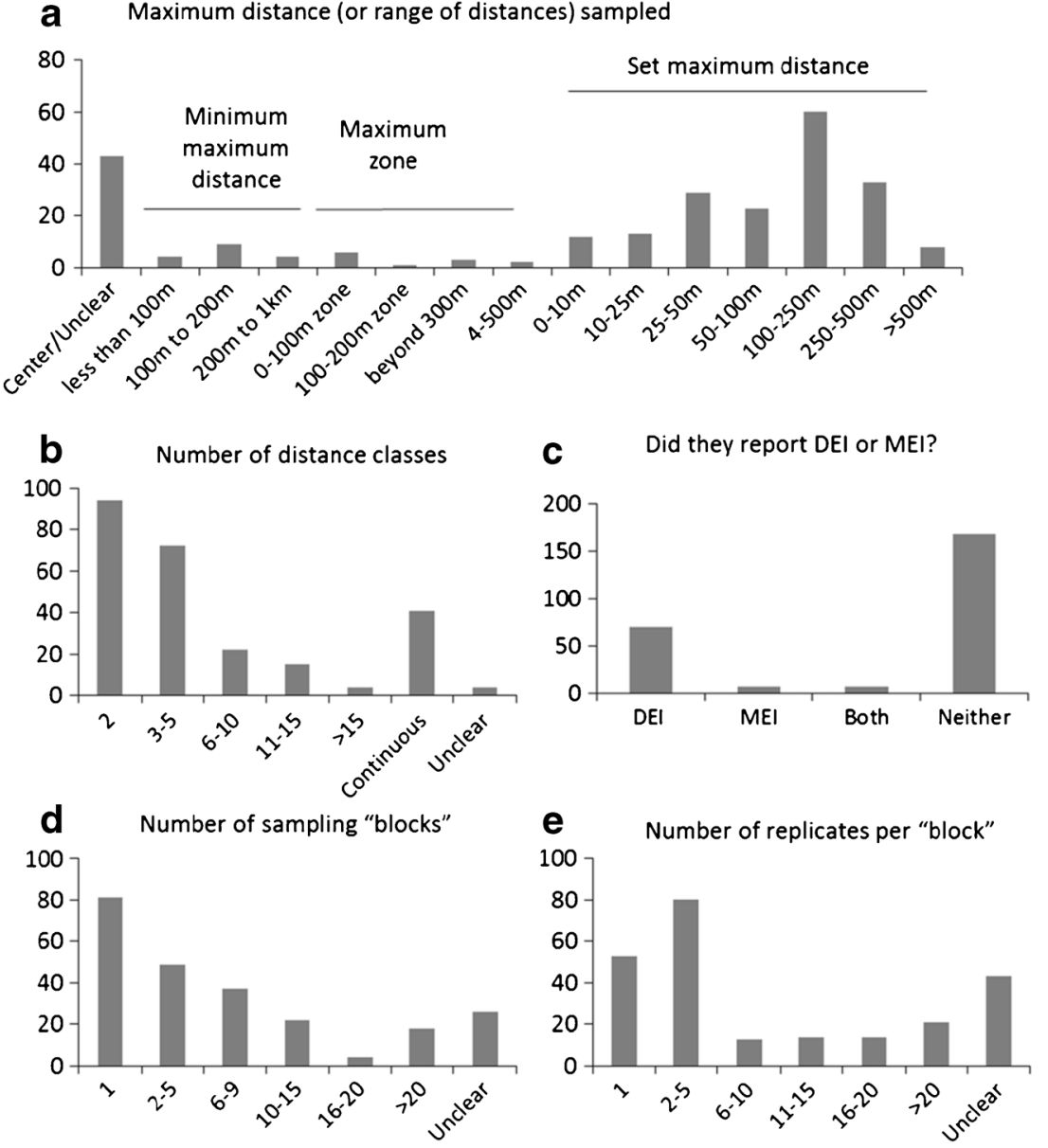

typically refers to the difference in response variables between the edge and interior areas of patches, but it can also be used to interpret effect sizes of response variables at different distances from edges [39]. These two components provide comprehensive understanding of the magnitude of edge effects and their influence across landscapes.

Quantifying MEI is important for determining the strength of edge effects and this measure requires identifying areas free of edge effects. Ewers and Didham [40] quantify the MEI as the difference in maximum and minimum values in a nonlinear logistic model, which can be determined from the first partial derivative of the model with respect to distance. Although care should be taken in interpretation because if the maximum and minimum occur in the two adjacent interiors respectively, the MEI may more closely reflect differences in habitat quality rather than edge to interior quality. Harper et al. [41] provide a standardized measure of MEI that scales the average responses at edge and interior locations such that MEI can range from -1 to 1 . This approach provides a useful means of quantifying MEI, with the added benefit of clear interpretations of effects across systems and response variables.

Quantifying DEI has proven more challenging than quantifying MEI and it has received considerable attention in recent years. Prior to 2000, the DEI was often visually determined [7]. Yet, formal methods now exist to quantify the DEI. Harper et al. [39] classified these methods into four categories: parametric, non-parametric, randomization, and curve-fitting (non-linear modeling hereafter) approaches. Parametric, nonparametric, and randomization approaches share similarities in the overall strategy for interpreting DEI but differ in the way in which statistical significance is determined. In these approaches, response variables are calculated as a function of distance from an edge (typically in binned categories of distance; e.g., 0-25 m, 26-50 m, etc.) and estimates are contrasted to interior locations. Note that the ability to capture the DEI can be limited based on study design if investigations do not sample at distances much greater than the underlying true DEI for the process of interest.

Nonlinear models can be fit to data as a function of distance from distance from edge (e.g., [40, 42-44]). Ewers and Didham [40] suggested a linear-logistic model to capture edge responses that occur across edge boundaries. In their model, the second partial derivative provides a measure of the inflection point on the curve, or an estimate of the depth of edge influence. Confidence intervals can then be calculated using bootstrapping. Hurst et al. [43] extended this approach to a 
mixed modeling framework, which accounts for spatial dependence along transects and can accommodate potential variation in the DEI over space or time through the use of random coefficients in the nonlinear model. Threshold or piecewise models have also been considered. Such models have the benefit of directly estimating inflection points in regression models and their associated uncertainty. Toms and Lesperance [45] provide an example of the application of this approach to edge effects.

While there has been an increase in the development of rigorous methods that can quantify the components of edge effects, most investigations still do not quantify DEI or MEI (Fig. 4c) because studies are still not designed to do so. For instance, the majority of studies from 2013-2015 still only have two distance classes (edge vs. interior, Fig. 4b) meaning that non-linear patterns like asymptotes are impossible to detect. Further, the maximum distance is often variable (meaning that you cannot pin down when the "interior" has been reached) or, even if it is set, the maximum distance is often less than $100 \mathrm{~m}$ (Fig. 4a) which may not reach a habitat's true interior. Because of this, only about $30 \%$ of recent studies characterized DEI and, even fewer (5\%), MEI (Fig. 4c). And of the $\sim 80$ studies that reported DEI, recommended techniques like randomization, piecewise regression, or the models by Ewers and Didham [40] were used only about $20 \%$ of the time. Unfortunately, there is still little agreement on the best approaches, although there is not necessarily any reason to converge on one best approach. Nevertheless, Harper et al. [39] argued that randomization methods were least sensitive to variation in study designs aimed at estimating edge effects, and, as such, advocated for their use in edgeeffect studies. Yet, those approaches do not provide formal estimates of DEI and their uncertainty; rather, DEI is inferred through post hoc comparisons of response variables near edges to interior. Further comparisons of these approaches, along with a set of 'best practices' for quantifying and reporting DEI and MEI, would help better guide research on edge effects.

\section{Extrapolating Edge Effects Across Landscapes}

There has been a long tradition in extrapolating edge effects across landscapes. Core area models provided the earliest approaches to extrapolating edge effects (e.g., [46-48]). Effective area models extended the core area concept to accommodate impacts of the matrix and variation in DEI and MEI [49]. These models have been proven useful in the extrapolation and mapping of potential edge effects across landscapes, but are rarely applied. Several challenges remain for extrapolating edge effects. These include: synergistic effects of multiple edges, the role of the matrix and landscape context, integrating edge effects with other components of fragmentation, and, finally, developing the tools to implement these priorities.

In real landscapes, effects from multiple edges (meaning the convergence of multiple habitats or complex geometry that causes edges to converge on themselves) can cause observed edge effects to be different than what would be expected from a single, straight edge. This issue, variously referred to as multiple edge effects [50] or edge-effect interactions [51], was originally formalized by Malcolm [52] but has been largely ignored. Yet, multiple edge effects can influence large areas of landscapes, up to $60 \%$ when edge responses are assumed to extend $120 \mathrm{~m}$ into habitat [53]. Even more importantly, a recent, comprehensive search of the edge literature found only 11 studies that had examined the issue closely; yet, strikingly, 10 out of 11 of those studies found support for multiple edge effects [51]. Further, in the papers we reviewed from 20132015, only one study considered these effects [54]. One potential barrier to this type of research is lack of tools. Quantitative methods that can quantify and extrapolate multiple edge effects have been proposed [52, 53, 55], but these methods need to become more user-friendly to provide broader applications.

Edge effects are impacted, indeed driven, by the structure of the matrix. This issue has long been acknowledged in investigations that focus on edge type and edge contrast, and the effective area model captures some types of matrix effects by allowing for edge responses to vary near different matrix types [49]. This inclusion has no doubt improved on the core area model, but is rarely used and other issues regarding matrix effects have not yet been considered. In addition to the issue of multiple edges (see above), Driscoll et al. [56] also emphasize that little is known regarding how the spatial extent of matrix composition surrounding patches (and not just adjacent to them) can influence edge effects.

Extrapolating edge effects across landscapes allows visualization of the role that edges may play in fragmented landscapes, yet as one scales up to landscapes several other issues can impact patterns and processes. Consequently, for landscape-scale modeling, edge extrapolation has been used less frequently than other types of modeling, such as species distribution [57] and connectivity modeling [58]. Species distribution modeling can accommodate multiple issues of relevance to patterns in fragmented landscapes, such as habitat composition and configuration, isolation, and edge effects (e.g., [59, 60]). Ultimately, edges are rarely accounted for in landscape-scale distribution models, even in those that focus on patch size and connectivity effects, despite the fact that edges are known to have a strong impact on local ecology.

Despite a lack of common use of edge-distance effects in larger models, one way that edges are incorporated into largescale models is by considering edge density (as measured in programs like Fragstats [61]), which is a measure of the length of edge per unit area. However, there has been little work 
connecting edge density specifically with different edge types and specific edge gradients (but see [59]). At present, it is difficult to connect the results of those studies to measurement of edge gradients at specific edge types for several reasons. First, edge density quantification rarely isolates edge density of certain types of edges thought to be critical for species distribution (but see, [62]) and are emphasized in models like the ERM. Second, it is unclear if edge density can appropriately capture non-linear and multiple edge effects observed in investigations on edge effects. Non-linear response and multiple edge effects suggest that edge density should not scale linearly with species responses in landscapes. Despite these issues, edge density is a tractable metric for large-scale investigations, especially in simple landscapes where a single focal habitat type is surrounded by a consistent type of matrix habitat (usually hostile). Further work that refines the concept of edge density to better capture known responses at habitat edges or determine what systems they are warranted in is needed.

\section{How Should Edge Studies be Designed?}

To advance and generalize our understanding of edge effects and the prediction of edge effects across landscapes, we make some general recommendations. Ultimately, the "right" study cannot be determined using design rules of thumb without knowing the specific question motivating research and knowledge of the individual study system. However, we offer some suggestions for study design that can hopefully allow researchers to avoid confounding edge effects with other factors and to avoid major problems of sample size. We address three issues: the number and type of edge categories and sample size.

For the number and type of categories, it is very important to consider the motivation of the study. If the goal of the study is to simply understand how edge and interior ecology differ from each other, then two edge categories (edge vs. interior) may be sufficient. However, if the goal is extrapolation, then ideally MEI and DEI should be measured at each unique (or comparable) edge type in the landscape and enough edge distance classes are needed to be able to determine if there is a threshold effect. Ultimately, this may take an iterative approach with many edge distances over a longer distance that can then be adjusted based on preliminary results. Sometimes, the maximum transect length possible will be limited by the amount of large remnants remaining. While logistics will often dictate the length and even the number of transects, there are some general rules that may be helpful. First, the most commonly detectable source of variability was edge orientation according to the 2004 review. To limit variability when sampling effort must be constrained, it may be useful to control for edge orientation and limit transects to edges most likely to show the steepest gradients (e.g., south-facing edges in the northern hemisphere). Second, avoid using broad distance categories (e.g., $>500 \mathrm{~m}, 10-100 \mathrm{~m}$, etc.). Ideally, edge categories should be consistent and each occur within a narrow band so they are more easily interpretable and comparable (both within and across studies).

For sample size, we emphasize having more independent sites rather than many sample units in a single or small number of sites. We suggest independent units be paired plots or transects rather than randomly arrayed plots at varying distance to account for local variability, which can be extreme. Over the last three years, the most common number of independent sampling sites was one, with remaining studies ranging from 2-15 (Fig. 4d). If no preliminary data are there to guide researchers, we suggest independent sampling units closer to ten. Also note that in edge studies, replication comes from the number of independent transects and not the number of distance classes. More distance classes allow better measurements of DEI and MEI but will not increase sample size. Carefully designed studies with sufficient sample size will promote results that can separate signals from noise and lend themselves to formal meta-analysis and comparison across studies. Increasing the number of studies that are rigorously comparable will allow edge research to progress and find generalities among widely divergent study systems and determine the theoretical frameworks that will allow us to make sense of highly variable systems.

\section{Conclusions}

After almost a century studying edge effects, we likely have as much information on how this one ecological factor influences distributions as almost any other across landscapes. Yet, we lag in using this information for landscape-scale studies compared to factors where, overall, less is known (e.g., connectivity) or that have proven unsuccessful in the past for understanding terrestrial fragmentation (e.g., island biogeography theory). Why is this? Part of it is certainly due to a tradition of designing edge studies only to measure qualitative aspects of edge effects, rather than in a way to apply them to landscape-scale analysis or to further conservation efforts. Unfortunately, typical experimental designs and/or low sample sizes will continue to stymie attempts to usefully apply study results and lead to a false sense that edge responses are hopelessly idiosyncratic. We advocate the continued study of empirical responses to edge effects for different species and different systems, especially if those studies are designed rigorously to measure DEI and MEI and are also placed into a conceptual framework that allows the inevitable variability of responses to be understood. We emphasize that in the future, efforts should be shifted towards developing and applying models that allow extrapolation to landscape scales. 
Acknowledgements We thank Lenore Fahrig and Matthew Betts for inviting us to write this review. This article was substantially improved by comments from Matthew Betts and two anonymous reviewers.

\section{Compliance with Ethical Standards}

Conflict of Interest Statement We have no conflicts of interest.

\section{References}

1. Clements FE. Plant physiology and ecology. New York: Holt; 1907.

2. Lahti DC. Why we have been unable to generalize about bird nest predation. Anim Conserv. 2009;12(4):279-81.

3. Alignier A, Deconchat M. Variability of forest edge effect on vegetation implies reconsideration of its assumed hypothetical pattern. Appl Veg Sci. 2011;14(1):67-74.

4. Villard MA. On forest-interior species, edge avoidance, area sensitivity, and dogmas in avian conservation. Auk. 1998;115(3):801-5.

5. Chalfoun AD, Thompson FR, Ratnaswamy MJ. Nest predators and fragmentation: a review and meta-analysis. Conserv Biol. 2002;16(2):306-18.

6. Vetter D, Rücker G, Storch I. A meta-analysis of tropical forest edge effects on bird nest predation risk: Edge effects in avian nest predation. Biol Conserv. 2013;159:382-95.

7. Ries L, Fletcher Jr RJ, Battin J, Sisk TD. Ecological responses to habitat edges: mechanisms, models, and variability explained. Annu Rev Ecol Evol Syst. 2004;31:491-522.

8. Macreadie PI, Connolly RM, Jenkins GP, Hindell JS, Keough MJ. Edge patterns in aquatic invertebrates explained by predictive models. Mar Freshw Res. 2010;61(2):214-8.

9. King DI, Griffin CR, DeGraaf RM. Effect of clearcut borders on distribution and abundance of forest birds in Northern New Hampshire. The Wilson Bulletin. 1997;1:239-45.

10. Fletcher Jr RJ, Koford RR. Spatial responses of bobolinks (Dolichonyx oryzivorus) near different types of edges in northern Iowa. Auk. 2003;120(3):799-810.

11. Ortega YK, Capen DE. Effects of forest roads on habitat quality for ovenbirds in a forested landscape. Auk. 1999;1:937-46.

12. Prevedello JA, Figueiredo MS, Grelle CE, Vieira MV. Rethinking edge effects: the unaccounted role of geometric constraints. Ecography. 2013;36(3):287-99.

13. Olson D, Andow D. Patch edges and insect populations. Oecologia. 2008;155(3):549-58.

14. Babak P, He F. A neutral model of edge effects. Theor Popul Biol. 2009;75(1):76-83.

15. Ruffell J, Didham RK. Towards a better mechanistic understanding of edge effects. Landsc Ecol. 2016;31:2205.

16. Villaseñor NR, Blanchard W, Driscoll DA, Gibbons P, Lindenmayer DB. Strong influence of local habitat structure on mammals reveals mismatch with edge effects models. Landsc Ecol. 2015;30(2):229-45.

17. Evans DM, Turley NE, Tewksbury JJ. Habitat edge effects alter antguard protection against herbivory. Landsc Ecol. 2013;28(9):174354.

18. Wimp GM, Murphy SM, Lewis D, Ries L. Do edge responses cascade up or down a multi-trophic food web? Ecol Lett. 2011;14(9):863-70.

19. Quevedo AA, Schleuning M, Hensen I, Saavedra F, Durka W. Forest fragmentation and edge effects on the genetic structure of Clusia sphaerocarpa and C. lechleri (Clusiaceae) in tropical montane forests. J Trop Ecol. 2013;29(04):321-9.

20. Laurance WF, Nascimento HE, Laurance SG, Andrade A, Ewers RM, Harms KE, et al. Habitat fragmentation, variable edge effects, and the landscape-divergence hypothesis. PLoS One. 2007;2(10): e1017.

21. Driscoll DA, Lindenmayer DB. Framework to improve the application of theory in ecology and conservation. Ecol Monogr. 2012;82(1):129-47.

22. Ries L, Sisk TD. Butterfly edge effects are predicted by a simple model in a complex landscape. Oecologia. 2008;156(1):75-86.

23. Villaseñor NR, Driscoll DA, Escobar MA, Gibbons P, Lindenmayer DB. Urbanization impacts on mammals across urban-forest edges and a predictive model of edge effects. PLoS One. 2014;9(5): e97036.

24. Ewers RM, Didham RK. Confounding factors in the detection of species responses to habitat fragmentation. Biol Rev. 2006;81(01): $117-42$.

25. Ries L, Sisk TD. A predictive model of edge effects. Ecology. 2004:85(11):2917-26.

26. Craig MD, Stokes VL, Hardy GE, Hobbs RJ. Edge effects across boundaries between natural and restored jarrah (Eucalyptus marginata) forests in south-western Australia. Austral Ecol. 2015;40(2): 186-97.

27. Ries L, Sisk TD. What is an edge species? The implications of sensitivity to habitat edges. Oikos. 2010;119(10):1636-42.

28. Fletcher Jr RJ, Ries L, Battin J, Chalfoun AD. The role of habitat area and edge in fragmented landscapes: definitively distinct or inevitably intertwined? Can J Zool. 2007;85(10):1017-30.

29. Bereczki K, Hajdu K, Báldi A. Effects of forest edge on pest control service provided by birds in fragmented temperate forests. Acta Zool Acad Sci Hung. 2015;61(3):289-304.

30. Robillard A, Johnson B. Edge effects on eastern massasauga rattlesnakes basking in managed habitat. Northeast Nat. 2015;22(1): 200-8.

31. Battin J. When good animals love bad habitats: ecological traps and the conservation of animal populations. Conserv Biol. 2004;18(6): 1482-91.

32. Martinson HM, Fagan WF. Trophic disruption: a meta-analysis of how habitat fragmentation affects resource consumption in terrestrial arthropod systems. Ecol Lett. 2014;17(9):1178-89.

33. Leopold A. Game management. New York: Charles Scribner's Sons; 1933. p. 481.

34. Alignier A, Alard D, Chevalier R, Corcket E. Can contrast between forest and adjacent open habitat explain the edge effects on plant diversity? Acta Botanica Gallica. 2014;161(3):253-9.

35. Crockatt ME. Are there edge effects on forest fungi and if so do they matter? Fungal Biol Rev. 2012;26(2):94-101.

36. Peyras M, Vespa NI, Bellocq MI, Zurita GA. Quantifying edge effects: the role of habitat contrast and species specialization. J Insect Conserv. 2013;17(4):807-20.

37. Comfort EJ, Clark DA, Anthony RG, Bailey J, Betts MG. Quantifying edges as gradients at multiple scales improves habitat selection models for northern spotted owl. Landsc Ecol. Online Early.

38. Ewers RM, Marsh CJ, Wearn OR. Making statistics biologically relevant in fragmented landscapes. Trends Ecol Evol. 2010;25(12): 699-704.

39. Harper KA, Macdonald SE. Quantifying distance of edge influence: a comparison of methods and a new randomization method. Ecosphere. 2011;2(8):1-7.

40. Ewers RM, Didham RK. Continuous response functions for quantifying the strength of edge effects. J Appl Ecol. 2006;43(3):52736

41. Harper KA, Macdonald SE, Burton PJ, Chen J, Brosofske KD, Saunders SC, et al. Edge influence on forest structure and composition in fragmented landscapes. Conserv Biol. 2005;19(3):768-82.

42. Zurita G, Pe'er G, Bellocq MI, Hansbauer MM. Edge effects and their influence on habitat suitability calculations: a continuous 
approach applied to birds of the Atlantic forest. J Appl Ecol. 2012;49(2):503-12.

43. Hurst ZM, McCleery RA, Collier BA, Fletcher Jr RJ, Silvy NJ, Taylor PJ, et al. Dynamic edge effects in small mammal communities across a conservation-agricultural interface in Swaziland. PLoS One. 2013;8(9):e74520.

44. Mancke RG, Gavin TA. Breeding bird density in woodlots: effects of depth and buildings at the edges. Ecol Appl. 2000;10(2):598611.

45. Toms JD, Lesperance ML. Piecewise regression: a tool for identifying ecological thresholds. Ecology. 2003;84(8):2034-41.

46. Temple SA, Cary JR. Modeling dynamics of habitat-interior bird populations in fragmented landscapes. Conserv Biol. 1988;2(4): 340-7.

47. Laurance WF. Edge effects in tropical forest fragments: application of a model for the design of nature reserves. Biol Conserv. 1991;57(2):205-19.

48. Laurance WF, Yensen E. Predicting the impacts of edge effects in fragmented habitats. Biol Conserv. 1991;55(1):77-92.

49. Sisk TD, Haddad NM, Ehrlich PR. Bird assemblages in patchy woodlands: modeling the effects of edge and matrix habitats. Ecol Appl. 1997;7(4):1170-80.

50. Fletcher RJ. Multiple edge effects and their implications in fragmented landscapes. J Anim Ecol. 2005;74(2):342-52.

51. Porensky LM, Young TP. Edge-effect interactions in fragmented and patchy landscapes. Conserv Biol. 2013;27(3):509-19.

52. Malcolm JR. Edge effects in central Amazonian forest fragments. Ecology. 1994;75(8):2438-45.

53. Li Q, Chen J, Song B, LaCroix JJ, Bresee MK, Radmacher JA. Areas influenced by multiple edges and their implications in fragmented landscapes. For Ecol Manag. 2007;242(2):99-107.
54. Porensky LM, Bucher SF, Veblen KE, Treydte AC, Young TP. Megaherbivores and cattle alter edge effects around ecosystem hotspots in an African savanna. J Arid Environ. 2013;96:55-63.

55. Fernández C, Acosta FJ, Abellá G, López F, Diaz M. Complex edge effect fields as additive processes in patches of ecological systems. Ecol Model. 2002;149(3):273-83.

56. Driscoll DA, Banks SC, Barton PS, Lindenmayer DB, Smith AL. Conceptual domain of the matrix in fragmented landscapes. Trends Ecol Evol. 2013;28(10):605-13.

57. Guisan A, Thuiller W. Predicting species distribution: offering more than simple habitat models (vol 8, pg 993, 2005). Ecol Lett. 2007;10(5):435.

58. Fletcher RJ, Burrell NS, Reichert BE, Vasudev D, Austin JD, Divergent perspectives on landscape connectivity reveal consistent effects from genes to communities. Curr Landscape Ecol Rep. 2016;1(2):67-79.

59. Betts MG, Fahrig L, Hadley AS, Halstead KE, Bowman J, Robinson WD, et al. A species-centered approach for uncovering generalities in organism responses to habitat loss and fragmentation. Ecography. 2014;37(6):517-27.

60. Fletcher RJ Jr, McCleery RA, Greene D, Tye C. Integrated models that unite datasets across scales reveal larger-scale environmental relationships and improve predictions of species distributions. Landsc Ecol. 2016;31:1369-82.

61. McGarigal K, Cushman SA, Ene E. FRAGSTATS v4: spatial pattern analysis program for Categorical and Continuous Maps. 4 ed. http://www.umass.edu/landeco/research/fragstats/fragstats. html2012.

62. Fletcher Jr RJ, Koford RR. Habitat and landscape associations of breeding birds in native and restored grasslands. J Wildl Manag. 2002;1:1011-22. 